\title{
LA LOTTA ALLE SUPERSTIZIONI: OBIETTIVI E DISCUSSIONI dal Libellus al Concilio di Trento
}

1. Tra i propositi di riforma che Giustiniani e Querini caldeggiarono nella lettera al nuovo pontefice Leone $\mathrm{X}$ vi era l'intento non secondario di sradicare gli atti, i riti e le credenze che gli autori definirono con il termine di 'superstizione.' Come si legge nel Libellus, si trattava di un flagello terribile che, associato con l'ignoranza, comportava gravi rischi di peccato mortale che avevano una precisa scaturigine: lo stato deplorevole del clero regolare e secolare, dal quale il popolo dei fedeli traeva cattivi esempi come "da una fonte" o da una "radice"; dall'alto al basso, per così dire. ${ }^{2}$ La superstizione è

${ }^{1}$ Per la storia del concetto di superstitio cf. Dieter Harmening, Superstitio. Überlieferungs- und theoriegeschichtliche Untersuchungen zur kirchlich-theologischen Aberglaubensliteratur des Mittelalters (Berlin: E. Schmitt, 1979); Girolamo Imbruglia, "Dalle storie di santi alla storia naturale della religione. Lidea moderna di superstizione," Rivista Storica Italiana 101 (1989): 34-84; Michael Bailey, "Concern over Superstition in Late Medieval Europe," in Past and Present 199, Supplement, 3, The Religion of Fools? Superstition Past and Present, ed. Stephen A. Smith and Alan Knight (Oxford: Oxford University Press, 2008), 115-33.

${ }^{2}$ Cito per comodità dalla traduzione italiana, non sempre efficace, di Geminiano Bianchini: Paulus Justiniani, Petrus Quirini, Lettera al Papa. Libellus ad Leonem $X$ [1513], a cura di Geminiano Bianchini, presentazione di Franco Cardini (Modena: Artioli, 1995), 89. Per il testo originale cf. Paulus Justiniani, Petrus Quirini, Libellus ad Leonem X, in Annales Camaldulenses Ordinis Sancti Benedicti tomus nonus, ed. Johannis-Benedicti Mittarelli et D. Anselmi Costadoni (Venezia: Giovanni Battista Pasquali, 1773), coll. 612-719, 675ss. Due stimolanti letture del testo in Adriano Prosperi, "Intellettuali e Chiesa all'inizio dell'età moderna," in Storia d'Italia, Annali 4: Intellettuali e Potere, ed. Corrado Vivanti (Torino: Einaudi, 1981), 159-252, in part. 177-252, e Giuseppe Alberigo, "Sul 'Libellus ad Leonem X' degli eremiti camaldolesi Vincenzo Querini e Tommaso Giustiniani," in Humanisme et Église en Italie et en France méridionale: XV. siècle-milieu du XVI. siècle, éd. Patrick Gilli (Rome: École Française, 2004), 349-59.

Franciscan Studies 71 (2013) 
"generatrice di tutti i mali,"3 avversaria della vera fede, peccato capitale contro la religione: idolatria. Fiorente come mai dopo la distruzione del paganesimo antico, tale colpa, agli occhi degli autori, avrebbe meritato una trattazione ben più estesa, al punto che i due eremiti dichiararono di volere mettere mano a "uno scritto più ampio." Ma per quanto ne so, un'opera de superstitione non venne mai pubblicata né da Querini, morto nel 1514, né da Giustiniani, che sarebbe vissuto fino al 1528. ${ }^{4}$ Del resto, il Libellus abbozzava già un progetto coerente di Reform before the Reformation - per usare il titolo di un libro piuttosto recente: ${ }^{5}$ un piano di reformatio della società cristiana che doveva essere guidato dalla $\mathrm{Cu}$ ria papale, dai vescovi e dal Concilio Laterano $\mathrm{V}$ voluto da Giulio II; ${ }^{6}$ e che, dopo la fase dell'emergenza protestante, e dopo la fine di un altro Concilio (quello di Trento, che come il precedente non avrebbe legiferato in materia di superstitio), sarebbe stato ripreso con linee analoghe nell'età della disciplina e della 'lotta contro le superstizioni'. ${ }^{7}$

3 “Ex hac ignorantia malorum omnium matre superstitio exoritur," Justiniani, Quirini, Libellus, col. 683.

${ }^{4}$ Justiniani, Quirini, Lettera al Papa, 107. Cf. Eugenio Massa, Una cristianità nell'alba del Rinascimento: Paolo Giustiniani e il 'Libellus ad Leonem X' (1513) (Genova: Marietti 1820, 2005), 151-52. Lo studioso riporta i contenuti di uno scritto sulle superstitioni stilato da Giustiniani intorno al 1513 per la sorella Lucia. Secondo Massa l'autore del Libellus non conobbe le pagine di Pomponazzi.

${ }^{5}$ Cf. Stephen D. Bowd, Reform before the Reformation. Vincenzo Querini and the Religious Renaissance in Italy (Leiden: Brill, 2002), in part. 193.

${ }^{6}$ Sul Laterano V si veda soprattutto Nelson H. Minnich, The Fifth Lateran Council (1512-17). Studies on his Membership, Diplomacy and Proposals for Reform (Aldershot: Variorum, 1993); Id., Councils of the Catholic Reformation: Pisa 1. (1409) to Trent (1545-63) (Aldershot: Ashgate Variorum, 2008). Una perorazione per la lotta contro le superstizioni in Antonio Pucci, Oratio habita in Nona Sacrosancti Lateranensis Concilii Sessione ([Roma]: [Silber], MDXIV). Un po' datato Silvio Tramontin, "Un programma di riforma della Chiesa per il Concilio Lateranense V: il 'Libellus ad Leonem X' dei veneziani Paolo Giustiniani e Pietro Quirini," in Venezia e $i$ Concili (Venezia: Fondazione Giorgio Cini, 1962), 67-95.

${ }^{7}$ Per l'Italia cf. soprattutto Giovanni Romeo, Inquisitori, esorcisti e streghe nell'Italia della Controriforma (Firenze: Sansoni, 1990); David Gentilcore, From Bishop to Witch: The System of the Sacred in Early Modern Terra D'Otranto (Manchester-New York: Manchester University Press, 1992); Adriano Prosperi, Tribunali della coscienza. Inquisitori, con- 
In che direzione? "Per sintetizzare - si legge nel Libellus - diciamo che [ogni superstizione] si riferisce sostanzialmente alla contraffazione di queste tre arti: della divinazione, della medicina e dell'artificiosa celebrazione di funzioni della nostra religione. Non c'è infatti nessuna città [...], nessuna casa, quasi nessuna mente di uomo, che [...] non sia in difficoltà per questi tre generi di superstizione." Gli autori della lettera pertanto denunciavano la diffusione delle pratiche di divinazione e dei libri di astrologia, invocandone la censura e il rogo; ${ }^{9}$ e puntavano il dito contro un certo naturalismo che scrutava nei cieli le sorti mondane degli uomini con tanto di favore dei principi. Inoltre, Querini e Giustiniani ribadivano che le cure non ufficiali in cui si impiegavano farmaci, sor-

fessori, missionari (Torino: Einaudi, 1996); Rainer Decker, Die Päpste und die Hexen. Aus den geheimen Akten der Inquisition (Darmstadt: Primus Verlag, 2003); Oscar Di Simplicio, Autunno della stregoneria. Maleficio e magia nell'Italia moderna (Bologna: il Mulino, 2005). Per i territori soggetti all'Inquisizione spagnola cfr. Gustav Henningsen, The Witches'Advocate. Basque Witchcraft and the Spanish Inquisition (Reno: University of Nevada Press, 1980); William Monter, Frontiers of Heresy: the Spanish Inquisition from the Basque Lands to Sicily (Cambridge: Cambridge University Press, 1990); Fernando Cervantes, Devils in the New World. The Impact of Diabolism in New Spain (New Haven-London: Yale University Press, 1997); María Tausiet Carlés, Ponzoña en los ojos. Brujería y superstición en Aragón en el siglo XVI (Zaragoza: Institución 'Fernando el Católico,' 2000); Maria Sofia Messana, Inquisitori, negromanti e streghe nella Sicilia moderna (1500-1782) (Palermo: Sellerio, 2007). Per il Portogallo e il suo Impero Laura de Mello e Souza, Inferno atlântico. Demonologia e colonização séculos XVI-XVIII (São Paulo: Companhia das Letras, 1993); José Pedro Paiva, Bruxaria e superstição num país sem "caça às bruxas" (1600-1774) (Lisboa: Notícias, 1997); Timothy D. Walker, Doctors, Folk Medicine and the Inquisition. The Repression of Magical Healing in Portugal during the Enlightenment (Leiden: Brill, 2005).

${ }^{8}$ Justiniani, Quirini, Lettera, 107. Cf. Id., Libellus, col. 684: Ut singula quaeque perstringamus, omnis potissimum ad harum trium artium imitationem refertur superstitio: ad divinationem, ad medicinam, \& ad affectatam caerimoniarum Religionis nostrae observantiam. Nulla enim est civitas [...], nulla domus, nulla pene hominis mens est, quae aliqua ex his tribus generibus superstitione non laboret.

${ }^{9}$ Justiniani, Quirini, Lettera, 108: "Piissimo padre [...], brucia tutti i libri degli astrologi, libri che riguardano la divinazione, condannati dalla Chiesa, dai Santi Concili e dai Santi Padri." Cf. Id., Libellus, col. 684: Astrologorum libros, qui ad divinationem pertinent, ab Ecclesia, a Sanctis Conciliis, Sanctisque Patribus damnatos omnes combure [Pientissime Pater]. 
tilegi e formule illecite non potevano ottenere la guarigione del paziente se non fuori dalle leggi di natura (non secundum propriam naturam). Si trattava in sostanza di mezzi diabolici rivolti contro la medicina e al tempo stesso contro la religione, che dovevano allearsi in una lotta comune contro i ciarlatani e i venditori di pomate e contro quegli scellerati che curavano senza riguardi alla medicina insegnata negli Studia.$^{10}$ Alla Chiesa spettava poi il compito più grave: quello di combattere, tra il clero e tra i fedeli, l'abuso di riti e di res sacrae, di preghiere, salmi e devozioni, di scongiuri e processioni, che offendevano il culto di Dio, la venerazione dei santi, la liturgia e un ortodosso modus orandi. ${ }^{11}$

Giorgio Caravale ha ricordato che il testo dei due camaldolesi segna un punto di partenza nella moderna strategia ecclesiastica di controllo e di riforma delle devozioni: una strategia che, se non avrebbe applicato, dopo la metà del XVI secolo, il consiglio di permettere ai fedeli la comprensione della Scrittura in volgare, almeno per l'uso che se ne faceva durante le celebrazioni, allo scopo di contrastare l'ignoranza del testo sacro, ${ }^{12}$ avrebbe ripreso molteplici aspetti della proposta del Libellus, compreso l'intento di fissare un rituale romano canonico. ${ }^{13} \mathrm{E}$ tuttavia - come si è visto - le pagine dedicate in quella lettera al peccato di superstitio prendevano di mira non solo il cattivo uso del culto, della liturgia e

${ }^{10}$ Cf. Justiniani, Quirini, Libellus, col. 685.

${ }_{11}$ Justiniani, Quirini, Lettera, 112: in nessun modo permetterai che siano esibiti salmi, dipinti, segni, amuleti, piccoli impiastri, brevi. Cf. Id., Libellus, coll. 686-87: psalmos, picturas, characteres, phlylacteria, pittaciola [sic], breves deferri nullo pacto permittes.

12 Justiniani, Quirini, Lettera, 105: "nulla è più necessario, più utile, più decoroso e conforme al comportamento dei Santi Padri che la traduzione dal latino in volgare di questi sacri testi che sono letti nella Chiesa." Cf. Id., Libellus, coll. 683: Nihil utilius omnino futurum, nihil magis decorum, atque secundum Sanctorum Patrum disciplinam institui posse, quam haec Sacrarum Scripturarum, quae in Ecclesia leguntur, ex Latino in vulgarem sermonem permutatio.

${ }^{13}$ Cf. Giorgio Caravale, Lorazione proibita. Censura ecclesiastica e letteratura devozionale nella prima età moderna (Firenze: Olschki, 2003), 1-7. Per la progressiva chiusura della Chiesa nei confronti della Scrittura in volgare cf. Gigliola Fragnito, La Bibbia al rogo. La censura ecclesiastica e i volgarizzamenti della Scrittura (1471-1605) (Bologna: il Mulino, 1997). 
della preghiera, ma anche il ricorso alle cure non approvate dalla medicina colta e la diffusione di scritti sugli astri e sul futuro. Il concilio provinciale fiorentino del 1517 avrebbe fatto propria, almeno sulla carta, l'istanza di una strenua lotta conto la superstizione magica diffusa tra i fedeli. ${ }^{14} \mathrm{Al}$ contrario, il Laterano V non avrebbe elaborato alcuna organica disposizione sulla materia, limitandosi a stigmatizzare sortilegi, invocazioni dei diavoli, incantesimi e divinazioni in un passo della Bulla de Reformatione Curiae riguardante il clero (sessione IX, 1514). ${ }^{15}$ Agli occhi dei padri riuniti a Roma, insomma, erano parsi più urgenti i pericoli della predicazione apocalittica e quello dell'empietà filosofica, tanto che quest'ultima ispirò la costituzione Apostolici regiminis del 19 dicembre $1513 .{ }^{16}$

2. E tuttavia la rinascita dell'averroismo e il naturalismo aristotelico ebbero molto a che fare con il nodo della superstizione, poiché negli stessi anni del Libellus dal mondo dei filosofi partì un attacco frontale verso alcune credenze di cui gli eremiti camaldolesi significativamente tacquero. Alludo a quel complesso di pratiche, riti e mitologie che avevano dato vita, nei due secoli precedenti, al 'concetto cumulativo di stregoneria.' Nel De incantationibus, che fu scritto nel 1520, dopo il Laterano V, e che ebbe grande circolazione manoscritta prima di essere stampato fuori d'Italia nel 1556, Pietro Pomponazzi pose l'accento sul fatto che il sistema di spiriti cristiano era inconciliabile con la metafisica e con la fisica dello Stagirita. Criticando la credenza nei morbi diabolici, egli interpretò convulsioni, patologie e miracoli 'soprannaturali' ricorrendo all'ipotesi 'fisica' per cui gli astri potevano condizionare la salute. Quel testo suscitò aspre reazioni anche perché vi si

${ }^{14} \mathrm{Cf}$. Romeo, Inquisitori, esorcisti e streghe, 187. Per gli intenti antisavonaroliani del sinodo cf. Stefano Dall'Aglio, L'eremita e il sinodo. Paolo Giustiniani e l'offensiva medicea contro Girolamo Savonarola, 1516-1517 (Firenze: Edizioni del Galluzzo, 2006).

${ }^{15}$ Cf. Conciliorum Oecumenicorum Decreta, ed. Giuseppe Alberigo et al. (Bologna: Dehoniane - Istituto per le Scienze Religiose, 1991), 625.

${ }^{16}$ Cf. in proposito Paola Zambelli, L’ambigua natura della magia, edizione rivista (Venezia: Marsilio, 1996), passim; Eric A. Constant, "A Reinterpretation of the Fifth Lateran Council Decree 'Apostolici Regiminis' (1513)," The Sixteenth Century Journal 33 (2002): 353-79. 
metteva in dubbio la collaborazione umana nei reati satanici (la nozione teologica di patto implicito o esplicito) e perché vi si negava il potere terapeutico degli scongiuri sacri. ${ }^{17} \mathrm{E}$ questo spiega il motivo per cui nell'Italia del Cinque e del Seicento sarebbe fiorita una letteratura per esorcisti che assolse al compito che nel Nord Europa fu rivestito dalla produzione di trattati teologici e giuridici che, dalla fine del XVI secolo in poi, avrebbe rinfocolato la paura nei demoni e nelle streghe, con esiti nefasti sul piano giudiziario. Gli scritti di Girolamo Menghi furono anche una risposta a Pomponazzi e ai suoi seguaci. ${ }^{18} \mathrm{E}$ tuttavia, già in anni vicini a quelli in cui fu stilato il Libellus, la diffusione della demonologia quattrocentesca aveva prodotto effetti anche in Italia. Il paradigma del sabba, infatti, fece vittime al di sotto delle Alpi nel feudo di Mirandola, dove Giovanni Francesco Pico, scettico e zelante filosofo e signore del luogo, nonché promotore di piani di riforma conciliari (un caso di savonarolismo intellettuale, l'ha definito Biondi ${ }^{19}$ ), incoraggiò Girolamo Armellini, inquisitore di Reggio e di Parma, a dare inizio a una caccia alle streghe che produsse una terribile scia di morti (1522-1525). Per rispondere alle critiche rivolte contro i processi Pico stilò il dialogo Strix, sive de ludificatione daemonum, poi volgarizzato dall'inquisitore Leandro Alberti. ${ }^{20} \mathrm{Ma}$ già in precedenza il tema della superstitio aveva fatto comparsa nelle pagine

${ }^{17}$ Cf. Pietro Pomponazzi, Gli incantesimi, a cura di Cristiana Innocenti (Firenze: La Nuova Italia, 1997), 39 (cap. 4), 132 (cap. 10). Ma vedi ora Id., De incantationibus, ed. Vittoria Perrone Compagni (Firenze: Olschki, 2011), 32, 105-06. Si rimanda all'edizione del testo e all'ampia introduzione della curatrice per la bibliografia. Per la fortuna dell'opera sempre utile Giancarlo Zanier, Ricerche sulla diffusione e fortuna del "De incantationibus" di Pomponazzi (Firenze: La Nuova Italia, 1975).

${ }^{18}$ Rinvio in proposito a quanto ho scritto in Vincenzo Lavenia, "Tenere i malefici per cosa vera.' Esorcismi e censura nell'Italia moderna," in Dal torchio alle fiamme. Inquisizione e censura, ed. Vittoria Bonani (Salerno: Biblioteca Provinciale, 2005), 129-72.

${ }^{19} \mathrm{Cf}$. Albano Biondi, Introduzione a Giovanfrancesco Pico della Mirandola, Strega o delle illusioni del demonio nel volgarizzamento di Leandro Alberti (Venezia: Marsilio, 1989), 9-41, 27.

${ }^{20}$ Cf. Ioannes Franciscus Picus, Dialogus in tres libros divisus: Titulus est Strix sive de ludificatione daemonum (Bologna: Hieronymo de Benedictis, 1523). Vedi anche Id., La sorcière. Dialogue en trois livres sur la tromperie des démons, éd. Alfredo Perifano (Turnhout: Brepols, 2007). 
del suo Examen vanitatis e nella misogina Oratio de reformandis moribus stilata nel 1514 per Leone X, pubblicata nel 1520 e poi riedita, in modo significativo, in appendice alla Strix. Se si guarda, inoltre, agli altri trattati coevi scritti in risposta a un nutrito partito di scettici che annoverava umanisti del calibro di Erasmo, fini giuristi come Andrea Alciati e Giovanni Francesco Ponzinibio e filosofi molto illustri quali Pomponazzi e Agrippa di Nettesheim, ${ }^{21}$ si ha l'impressione che la lotta contro le superstizioni, negli anni immediatamente successivi alla stesura del Libellus, si risolvesse in una battaglia orientata ad affermare la realtà del sabba e del volo notturno e a confutare una tradizione normativa e teologica sulla superstitio che, fino dal Canon Episcopi, aveva rubricato certe credenze come mere illusioni o reliquie di paganesimo. ${ }^{22}$ Vero è che religiosi quali l'inquisitore che precedette frate Bartolomeo Spina come giudice della fede di Modena parvero dare seguito alle istanze del Libellus, aprendo processi contro chierici scandalosi e 'superstiziosi' come il pittoresco Guglielmo Campana studiato da Matteo Duni. ${ }^{23}$ Tuttavia la trattatistica circolata dopo il Concilio Laterano V sembra orientata in una direzione molto più precisa.

Basti elencare le opere scritte dai frati predicatori Silvestro Mazzolini, Bernardo Rategno e Spina stesso. Il primo, nel 1521, stilava i De strigimagarum demonunque mirandis libri tres ${ }^{24}$ il secondo il Tractatus de strigiis e una Lucerna

${ }^{21}$ Rimando in proposito, anche per la bibliografia più aggiornata, a Matteo Duni, "La caccia alle streghe e i dubbi di un giurista. Il 'De lamiis et excellentia utriusque iuris' di Giovan Francesco Ponzinibio," in La centralità del dubbio. Un progetto di Antonio Rotondò, ed. Camilla Hermanin e Luisa Simonutti, 2 vol. (Firenze: Olschki, 2011) I, 3-26; Id., "I dubbi sulle streghe," in I vincoli della natura. Magia naturale e stregoneria nel Rinascimento, ed. Germana Ernst e Guido Giglioni (Roma: Carocci, 2012), 203-221.

${ }^{22}$ Cf. L'imaginaire du Sabbat. Édition critique des textes les plus anciens, éd. Martine Ostorero et al. (Lausanne: Université de Lausanne, 1999).

${ }^{23}$ Cf. Matteo Duni, Tra religione e magia. Storia del prete modenese Guglielmo Campana (1460?-1541) (Firenze: Olschki, 1999).

${ }^{24}$ Sull'autore cf. Michael Tavuzzi, Prierias. The Life and Works of Silvestro Mazzolini da Prierio (1456-1527) (Durham, N.C.-London: Duke University Press, 1997). 
inquisitorum ${ }^{25}$ il terzo, nel 1523, la Quaestio de strigibus, a cui avrebbero fatto seguito le Apologiae in Ponzinibium de lamiis. Si trattava di volumi destinati a dimostrare la realtà del volo notturno e la necessità della caccia alle streghe. Il Libellus, al contrario, non aveva affatto alluso al sabba, non aveva riservato se non un cenno ai malefici e aveva orientato la lotta contro le superstizioni in una direzione diversa: la disciplina dei riti e delle devozioni, il divieto di scrivere di divinazione e di praticare l'astrologia, il controllo delle pratiche terapeutiche non ufficiali e concorrenti con la medicina colta e con gli esorcismi della Chiesa. Si trattò di una posizione isolata? E fu, quella del Libellus, una linea perdente, prima del Tridentino? Fu una proposta precoce, che avrebbe avuto attuazione solo molti anni dopo, con la bolla Coeli et Terrae di Sisto V? ${ }^{26}$ E che rapporto esiste tra i discorsi più 'mitologici' sulla stregoneria e quelli più generali sulla superstitio negli anni centrali del Cinquecento? Ha ragione Stuart Clark, quando scrive che i progetti di riforma di tutte le pratiche e dei riti non ortodossi e i piani repressivi dei demonologi più ossessionati dal complotto delle streghe finirono di fatto per sovrapporsi in nome della nozione di eresia e della catalogazione di ogni superstizione, semplice e diabolica, come un peccato contro il primo comandamento? ${ }^{27}$ Infine, si può concordare con John Bossy quando osserva che, sul piano disciplinare, la centralità acquistata dal decalogo, in virtù dell'influsso del nominalismo e del magistero di Jean Gerson, produsse una vera e propria ossessione del clero e dei fedeli verso i peccati che violavano il primo comandamento, con effetti quanto meno indiretti sulla caccia alle streghe? ${ }^{28}$ $\mathrm{O}$ è possibile dire il contrario: che trattare più complessiva-

${ }^{25}$ Cf. Tamar Herzig, "Rategno, Bernardo of Como," in Encyclopedia of Witchcraft: The Western Tradition, ed. Richard M. Golden, 4 vol. (Santa Barbara: ABC-CLIO, 2006), 951-52.

${ }^{26} \mathrm{Cf}$. Romeo, Inquisitori, esorcisti e streghe.

${ }^{27}$ Cf. Stuart Clark, Thinking with Demons. The Idea of Witchcraft in Early Modern Europe (Oxford: Oxford University Press, 1996), 485-88.

${ }^{28}$ Cf. John Bossy, "Moral Arithmetic: Seven Sins into Ten Commandments," in Conscience and Casuistry in Early Modern Europe, ed. Edmund Leites (Cambridge: Cambridge University Press, 1988), 214-34, trad. it.: "Aritmetica morale: sette peccati in dieci comandamenti," in John Bossy, 
mente delle violazioni del primo comandamento (e dunque di superstitio) comportò lo spostamento di attenzione dal maleficio alla credenza nei poteri del diavolo, permettendo così di trattare la stregoneria come un'eresia castigabile nei limiti del ius canonicum (che non prevedeva il rogo per il colpevole primo lapsu) e riducendo l'importanza attribuita, sul piano giudiziario, al danno materiale che era la fonte di gran parte delle accuse contro le streghe? Sappiamo da tempo che nella seconda metà del Cinquecento il dibattito sul sabba e i processi in cui compare il volo notturno conobbero un declino nelle aree nell'Europa cattolica controllate dalle Inquisizioni centralizzate moderne. ${ }^{29}$ La lotta alle superstizioni, per sintetizzare, non si accompagnò necessariamente all'apertura di processi per stregoneria sabbatica anche in presenza di accuse di maleficio (e nonostante abusi e condanne a morte che pure ci furono, specie nel Nord Italia e negli anni di Carlo Borromeo). ${ }^{30} \mathrm{Ma}$ forse occorre analizzare meglio anche l'arco di tempo che va dal Laterano $\mathrm{V}$ al Tridentino, perché il dibattito sul sabba (ben noto agli storici) ha forse oscurato un'ampia elaborazione teologica sulla superstitio e le violazioni contro il primo comandamento che andò in una direzione diversa rispetto alla demonologia vera e propria, talvolta con espliciti intenti polemici e non senza venature scettiche. Il Libellus, in questo senso, mi pare uno snodo significativo: in tempi di intenso dibattito, gli autori tacevano di streghe e di voli ma invocavano il controllo dei riti e delle pratiche del clero e dei fedeli. Furono gli stessi anni in cui si fece evidente una divisione che attraversò anche l'Ordine dei frati predicatori: quei Domini canes impegnati nell'Officium fidei e membri della famiglia religiosa da cui provenivano giudici come l'autore del Malleus. ${ }^{31}$

$\overline{\text { Dalla comunità all'individuo. Per una storia sociale dei sacramenti nell'Eu- }}$ ropa moderna, introd. Adriano Prosperi (Torino: Einaudi, 1998), 87-116.

${ }^{29}$ Vedi la bibliografia citata supra alla nota 7.

${ }^{30}$ Cf. Vincenzo Lavenia, "Stregoneria, Italia," in Dizionario storico dell'Inquisizione, ed. Adriano Prosperi, Vincenzo Lavenia e John Tedeschi (Pisa: Edizioni della Normale, 2010) III, 1521-530.

${ }^{31}$ Per ricostruire i contatti e i percorsi di alcuni demonologi e inquisitori tra la fine del Quattrocento e l'inizio del Cinquecento (e in particolare per Heinrich Kramer) cf. Tamar Herzig, "Bridging North and South: In- 
3. Per comprendere tale divisione, è forse utile guardare alla prima delle tre opere che ho scelto di prendere in esame. Si tratta del commento più autorevole e diffuso di sempre alla Summa Theologiae di Tommaso, il testo che lentamente, nel corso del Cinquecento, avrebbe sostituito le Sententiae di Pietro Lombardo come 'manuale' di base per la formazione nelle Scholae. L'Aquinate aveva trattato di superstizione nelle quaestiones 91 e seguenti della Secunda Secundae; e fu chiosando quelle pagine (in cui era stata elaborata la sottile nozione di 'patto implicito') che nel 1518 il frate Tommaso De Vio, meglio noto come cardinale Gaetano, espresse la propria posizione subito dopo la chiusura del Laterano V, attirandosi per questo le velate repliche polemiche di confratelli rivali come Mazzolini e Spina. Creduto «in imaginatione», scrisse il Gaetano, il sabba di cui tanto si parlava da qualche tempo (soprattutto al di là delle Alpi) era frutto di malattia mentale o dell'illusione di povere vecchie dai nervi compromessi; e lo dimostrava l'esperienza di saggi giudici che avevano rinchiuso donne inquisite che avevano testimoniato comunque di voli notturni mai avvenuti 0 , chissà, evocati in sogno grazie all'impiego di allucinogeni. "Non negamus" - continuò De Vio - che "Deo permittente" il volo si possa verificare "etiam corporaliter," ma tuttavia "rarissime." Il Canon Episcopi, ai suoi occhi, manteneva dunque quasi intatto il suo valore, e l'emergenza più seria che si poneva di fronte alla Chiesa era la stessa individuata pochi anni prima nel Libellus. De Vio, infatti, osservò che il terreno di indagine più utile per la disciplina dei fedeli - e per un esercizio più esteso della giurisdizione degli inquisitori - era fornito non tanto dalla caccia alle streghe, quanto piuttosto dalle diffuse pratiche superstiziose e dai sortilegi, che avevano risvolti ereticali anche in assenza del patto esplicito con i diavoli, "pro sociali actu

quisitorial Networks and Witchcraft Theory on the Eve of the Reformation," Journal of Early Modern History 12 (2008): 361-82. Cf. Hans Broedel, The 'Malleus Maleficarum' and the Construction of Witchcraft. Theology and Popular Belief (Manchester-New York: Manchester University Press, 2003). Per la celeberrima opera di Institor cf. Heinrich Kramer, Der Hexenhammer. Malleus Maleficarum, ed. Wolfgang Behringer, Günter Jerouschek und Werner Tschacher (München: Deutscher Taschenbuch, 2004). 
cum daemone": cioè in virtù della pratica stessa, che implicava l'“invocatio tacita" del Maligno. Anche se formalmente non ereticali, certi atti e certe formule si rivolgevano sempre ai diavoli: "materialiter ad cultum eorum spectant," "quamvis non fiant tali animo." "Accipe dicta theologice," concluse il Gaetano: non intendo parlare di competenze giudiziarie; non intendo suggerire che gli "inquisitores procedant" contro le mere e semplici superstizioni: "gaudet unaquaeque scientia suis terminis," si schermì. ${ }^{32}$ E tuttavia un uomo autorevole, allora generale dei frati predicatori (sebbene non gradito a chi ne denunciava la presunta vicinanza a Erasmo e agli empi filosofi averroisti), più tardi teologo 'ufficiale' sulla linea di Tommaso, aveva proposto una strategia non distante da quella prospettata nel Libellus. E tale strategia, per un paradosso, avrebbe avuto rapida applicazione proprio nella terra in cui le dottrine del cardinale De Vio furono meno amate: cioè in quella Spagna in cui da tempo faceva le sue prove il tribunale centralizzato dell'Inquisizione. Un foro che - com'è noto - non fu impiantato per combattere le pratiche di sortilegio o di divinazione, la medicina illecita e l'abuso dei riti; ma che con le superstizioni e la paura delle streghe dovette fare i conti prima che nascesse il Sant'Uffizio papale.

4. Nei primi quarant'anni di vita la macchina giudiziaria voluta da Fernando e Isabela agì soprattutto contro i conversos; ma più tardi si trattò di affrontare anche le derive mistiche degli alumbrados, l'eresia 'luterana,' l'erasmismo, la superstizione e la stregoneria. Nel 1525, nella regione pirenaica della Navarra, fu intrapresa una campagna che costrinse i vertici dell'Inquisizione a occuparsi di un'ondata di panico che aveva identificato centinaia di bersagli. Pertanto nel 1526 una junta di teologi e giuristi, riunita a Granada, fu chiamata a pronunciarsi sulle carte arrivate alla Suprema. A quel punto, pur con sfumature diverse e non senza contrasti,

${ }^{32}$ Cito da Thoma de Aquino, Secunda secundae partis Summae Theologiae [...] cum commentariis [...] Thomae De Vio Caietani (Venezia: apud Iuntas, 1588), col. 234rff. Ha ricostruito la fortuna delle opere teologiche del Gaetano dopo la fondazione del Sant'Uffizio romano Claus Arnold, Die römische Zensur der Werke Cajetans und Contarinis (1558-1601). Grenzen der theologischen Konfessionalisierung (Paderborn: Schöningh, 2008). 
il dibattito si orientò in una direzione 'scettica.' Non si negò che il sabba potesse verificarsi, ma si ritenne che la pena di morte non fosse il castigo più adeguato per la superstizione, e che i vertici del tribunale dovessero dire l'ultima parola sulle sentenze emesse a livello locale. Inoltre se un imputato dichiarava di avere visto qualcuno al sabba, la chiamata di correo doveva destare sospetti per la capacità del demonio di illudere le menti. Si volle interrompere in questo modo il meccanismo che portava a moltiplicare le accuse e si prescrisse di accertare che gli imputati si fossero mossi realmente dai loro giacigli, anche quando lo avessero testimoniato senza tortura. Le istruzioni di Granada, come ha sottolineato Monter, segnarono una svolta e suggerirono una linea dalla quale i vertici del tribunale non si sarebbero più discostati; inoltre la ricerca sulle fonti d'archivio ha confermato che almeno in Castiglia (nei regni di Aragona e nel Nuovo Mondo la storia fu più complessa) nel corso dell'età moderna il controllo della Suprema sulle cause locali risultò efficace. I giudici della fede, insomma, vollero combattere soprattutto la superstitio e i sortilegi, e non i riti notturni. ${ }^{33}$

Fu qualche anno dopo le deliberazioni della junta che apparvero due testi in spagnolo dedicati entrambi ai peccati di superstizione. Il primo, il Tratado de las superstitiones y hechizerias, era opera di Martín de Castañega, un frate minore di Burgos che prese posizione contro le illusioni sabbatiche delle streghe e fece pubblicare il libro a Logroño, nei Paesi Baschi, da Miguel de Eguía (1529). ${ }^{34}$ L'altro, la cui prima edizione risale forse al 1530, era frutto dell'impegno di un cattedratico che insegnava ad Alcalá de Henares e aveva partecipato alla junta che nel 1527 a Valladolid era stata incari-

${ }^{33} \mathrm{Cf}$. Monter, Frontiers of Heresy, 259-63.

${ }^{34} \mathrm{Il}$ testo è stato ripubblicato più volte anche nel Novecento: Martín de Castañega, Tratado de las supersticiones y hechicerías y de la posibilidad y remedio dellas (1529), ed. Juan Robert Muro Abad (Logroño: Instituto de Estudios Riojanos, 1994). Cf. David Darst, "Witchcraft in Spain: The Testimony of Martín de Castañega's Treatise on Superstition and Witchcraft (1529)," Proceedings of the American Philosophical Society 123 (1977): 298322; María Tausiet Carlés, "Religión, ciencia y superstición en Pedro Ciruelo y Martín de Castañega," Revista de Historia Jerónimo Zurita 64-65 (1992): 139-48; Clark, Thinking with Demons, 487. 
cata di vagliare l'ortodossia dell'opera di Erasmo. Si trattava di Pedro Ciruelo e lo scritto - finemente analizzato anche in anni recenti ${ }^{35}$ - si intitola Reprobación de las supersticiones $y$ hechicerías. Bataillon ha liquidato Ciruelo, autore di un confessionario, come un filosofo di scarso spessore, ${ }^{36}$ ma in realtà si trattava di un converso influenzato dalla linea teologica nominalista e da Gerson (si era formato a Parigi) e che poteva vantare di avere scritto diverse pagine per confutare l'astrologia giudiziaria e le tesi di Pomponazzi. In più, il suo trattato non era una novità nella Spagna del tempo. Senza contare il testo di Castañega, basti dire che nel 1510, contro la paura delle streghe diffusa nell'area pirenaica, il canonico navarro Martín de Andosilla (o di Arles) aveva pubblicato un libro De superstitionibus ispirato soprattutto al De erroribus circa artem magicam di Gerson. ${ }^{37}$ Anche in quel testo si dubitava del volo, ma si dichiarava reale, come nel Malleus, il coito tra i demoni e le streghe.

Ciruelo, come già Andosilla, accennò in alcuni passi dell'opera alla credenza nel volo notturno, ma senza insistervi. Il suo scopo, infatti, era ancora una volta quello di classificare i peccati contro il primo comandamento sulla scia di quanto avevano fatto prima di lui Gerson, Alonso de Espina, Andosilla, Giustiniani e Querini. ${ }^{38}$ Tuttavia una differen-

${ }^{35}$ Cf. Lu Ann Homza, Religious Authority in the Spanish Renaissance (Baltimore-London: The Johns Hopkins University Press, 2000), 176-209.

${ }^{36} \mathrm{Cf}$. Marcel Bataillon, Érasme et l'Espagne, II éd. texte établi par Daniel Devoto, éd. Charles Amiel, 3 voll. (Genève: Droz, 1991) I, 83.

${ }^{37}$ Cf. Martinus de Arles de Andossilleve, Tractatus percelebris et ad maleficia, superstitionesque tollendas oppido quam necessarius (Lyon: per Ioannem Cleyn, 1510). Negli anni di Gregorio XIII l'opera meritò di essere inclusa nel Tractatus Universi Iuris (Venezia: ad signum Aquilae se renovantis, 1584) XI/2, f. 402ss, ma era stata stampata anche a Parigi (1517), Roma (1559) e Francoforte (1581). Per la biografia dell'autore e un'edizione moderna del testo (ripubblicato e tradotto in castigliano di recente, nel 2011) cf. José Goñi Gaztambide, "El tratado "De superstitionibus" de Martín de Andosilla," Cuadernos de Etnología v Etnografía de Navarra 3 (1971): 249-332.

${ }^{38}$ Cf. Pedro Ciruelo, Tratado en el qual se repruevan todas las supersticiones y hechizerias con nuevas adiciones a cada capitulo del dotor Pedro Antonio Iofreu (Barcelona: Sebastian de Cormellas, 1628). I passi sul sabba 19-20, 46-47, dove l'autore rimette il giudizio su quei racconti ai giudici dell'Inquisizione. Si preferisce citare da questa edizione invece che dalla 
za rilevante fu la sua scelta di scrivere in volgare un'opera piuttosto agile e complessa che meritò di essere ripubblicata ancora nel Seicento - nel tempo in cui in Europa si moltiplicavano i roghi - con l'aggiunta di una significativa difesa del Canon Episcopi contro i novatores che asserivano con fanatismo la realtà del volo notturno delle streghe (l'apologia era opera di un giurista catalano, Pedro Antonio Iofreu, e venne approvata dall'Inquisizione di Barcellona) ${ }^{39} \mathrm{Il}$ motivo per cui l'opera di Ciruelo servì in quel contesto come antidoto alla credenza del sabba lo si comprende leggendo il testo, dove le colpe contro il primo comandamento erano definite le più gravi perché «mas desplazen a Dios y mas daño hazen a los hombres». ${ }^{40}$ L'autore, del resto, polemizzò contro il determinismo astrologico (negare il diavolo e credere alle stelle significava negare la fede e l'anima), difese la nozione di patto implicito, attaccò il peccato di idolatria, ma soprattutto puntò, come il Libellus, sulla riforma delle pratiche mediche e dei sacri scongiuri. Come i camaldolesi, Ciruelo era convinto che la superstitio fosse un cattivo habitus che si trasmetteva dall'alto al basso, dal clero ai fedeli. E perciò esortò la Chiesa (e l'Inquisizione) al controllo dei rituali e degli esorcismi, al vaglio dei fenomeni rubricati come possessioni demoniache (erano gli anni in cui si diffondeva il fenomeno delle beatas), ${ }^{41}$ alla vigilanza sulla medicina popolare e sui sortilegi, alla di-

ristampa anastatica dell'edizione di Salamanca del 1538 (Madrid 1952, nuova edizione nel 2003 con introduzione di José Luis Herrero Ingelmo), dall'edizione di Valencia del 1978 (priva di marginalia) e dalla traduzione inglese, che del resto è condotta sul testo del 1628 ed è apparsa negli Usa nel 1977. Una traduzione tedesca è quella curata da Mariano Delgado (2008).

${ }^{39}$ Cf. Pedro Antonio Iofreu, "Defensa del Canon Episcopi 26. quaestio n. 5," in Ciruelo, Tratado, 251-64.

${ }^{40}$ Ciruelo, Tratado, 17.

${ }^{41}$ Ciruelo, Tratado, 178ss. Sul fenomeno religioso cf. Adelina Sarrión Mora, Beatas y endemoniadas. Mujeres heterodoxas ante la Inquisición, siglos XVI a XIX (Madrid: Alianza, 2003); Alison Weber, "The Inquisitor, the Flesh, and the Devil: Alumbradismo and Demon Possession," in Dämonische Besessenheit. Zur Interpretation eines kulturhistorischen Phänomens, ed. Hans de Waardt et al. (Bielefeld: Verlag für Regionalgeschichte, 2005), 117-91. 
sciplina delle orazioni e delle devozioni, perché Dio, fece osservare, non opera i miracoli «cadaldia y hora». ${ }^{42}$

Quanti curavano senza avere appreso la medicina ufficiale agivano come finti santi e facevano concorrenza agli esorcismi, che dovevano essere somministrati secondo formule approvate e senza inventare nuove giaculatorie a danno alle anime. Gli esorcisti inoltre dovevano evitare di interrogare i diavoli e impedire gli spettacoli pubblici di scongiuro, perché il diavolo era il padre della menzogna e poteva fingere possessioni e malefici. ${ }^{43}$ Inoltre fu proprio Ciruelo, a quanto mi è dato di sapere, il primo a suggerire una norma di grande rilevanza adottata più tardi nei processi del Sant'Uffizio: quella di imporre il consulto dei medici prima di dichiarare vera una malattia diabolica in sede di giudizio. ${ }^{44} \mathrm{E}$ tale posizione mette in luce come il progetto di riforma elaborato da $\mathrm{Ci}$ ruelo per combattere le superstizioni fosse in sintonia con le strategie ecclesiastiche e inquisitoriali poi praticate in epoca post-tridentina. ${ }^{45}$ Insomma, l'ossessione per i peccati contro il primo comandamento, come già nel Libellus, ebbe come esito la stesura di un testo in cui la demonologia ricopriva uno spazio del tutto marginale a vantaggio dell'auspicato controllo della medicina, della divinazione e dei riti ecclesiastici.

5. Ma anche la rigorosa posizione tomistica di De Vio non rimase isolata. Molto si è scritto sulle relectiones di Francisco de Vitoria dedicate alla guerra e agli indios; ma scarsa attenzione ha suscitato una lezione che il grande teologo di Salamanca dedicò nel 1540 alla magia (fu pubblicata, come le altre, nel 1557, post mortem, grazie agli appunti degli allievi). ${ }^{46}$ Commendando un passo del Levitico dedicato al castigo della pratiche magiche, anche Vitoria insinuò che il

${ }^{42}$ Ciruelo, Tratado, 125.

${ }^{43}$ Ciruelo, Tratado, 183-85.

44 “Tomen informacion del sabio medico," suggerisce Ciruelo alle autorità religiose in tema di morbi diabolici. Ciruelo, Tratado, 185.

${ }^{45} \mathrm{Mi}$ permetto di rinviare a Vincenzo Lavenia, La medicina dei diavoli: il caso italiano, secoli XVI-XVII, in Médicine et religion. Collaborations, compétitions, conflits (XIIe-XXe siècle), éd. M. P. Donato et al (Roma: École Française, 2013), 163-94.

${ }^{46}$ Cf. Franciscus de Vitoria, Relectionum Theologicarum secundus tomus (Lyon: apud Boyerum, 1557), 248-318 (De Arte Magica). Una tradu- 
volo delle "bruxas" o "lamias" o "striges" fosse illusorio, e per dimostrarlo riferì della polemica che aveva investito nel 1505 la Quaestio lamiarum del frate minore italiano Samuele Cassini, uno scotista antisavonaroliano nemico dell'uso politico e femminile della profezia contro il quale avrebbe impugnato la penna Pico stilando una Defensio del frate predicatore ferrarese e contro cui il frate domenicano Vincenzo Dodi scrisse una Apologia contra li defensori delle strie che meritò una replica di Cassini (1506). ${ }^{47}$ Vitoria (un domenicano) polemizzò tuttavia in modo diretto non con i confratelli demonologi, ma con quei filosofi peripatetici che accusava di mettere in dubbio l'esistenza del diavolo e il patto demoniaco (e non solo il sabba, a cui Vitoria era disposto a rinunciare) per porre l'accento sull'utilità civile del timore (cioè sull'impostura delle religioni). Persino gli indios, osservò, nulla sapendo delle aberrazioni degli averroisti, credevano nelle forze del male senza avere ricevuto il lume della rivelazione. ${ }^{48}$ Vitoria inoltre citò ripetutamente il Canon Episcopi e negli stessi anni si occupò dei peccati di superstitio commentando, come già De Vio, la Secunda Secundae (le lezioni sarebbero rimaste manoscritte fino al Novecento). In quel contesto tentò una classificazione di quel genere di peccati prestando attenzione alla pratica della confessione; e sulle streghe ribadì quanto scrisse nella Relectio. La disputa sul volo, disse, agita soprattutto gli italiani e aveva visto contrapposti i canonisti, in genere scettici, ai teologi, convinti invece della realtà delle tesi di Johannes Nider. Vitoria non si sbilanciava ma si limitava a citare un eloquente racconto di Alonso Madrigal (più noto come il Tostado), che nei suoi commenti scritturali aveva riferito di una donna convinta di volare dopo essersi unta e

zione moderna in Id., Sobre la magia, ed. Luis Frayle Delgado (Madrid: Tecnos, 2006).

${ }^{47}$ Vitoria, De Arte Magica, 257-58, 309-10. Su Cassini e su Dodo cf. le voci di Michaela Valente in Encyclopedia of Witchcraft, 172-73, 287-88; sulla polemica tra Cassini e i seguaci di Savonarola cf. Tamar Herzig, $S a$ vonarola's Women. Visions and Reform in Renaissance Italy (Chicago and London: The Chicago University Press), 2008, ad indicem.

${ }^{48} \mathrm{Cf}$. Vitoria, De Arte Magica, 273. 
rimasta ferma nel suo letto sotto gli occhi di un teologo che aveva criticato il mito del sabba. ${ }^{49}$

6. I peccati di superstizione ebbero uno spazio crescente anche nelle summae per i confessori della prima metà del XVI secolo: da quella dell'inquisitore di Bologna Giovanni Cagnazzo (la Tabiena, nella quale compare un riferimento al Malleus in un lungo passo che riporta notizie dei roghi del Nord Italia, mostra dubbi sulle presunte metamorfosi animali delle streghe ma di fatto accetta l'idea della realtà del volo notturno) a quella del giudice della fede di Piacenza Bartolomeo Fumi (l'Aurea Armilla), che dedicherà un contraddittorio lemma specifico al problema delle streghe (entrambi gli autori erano frati predicatori). ${ }^{50} \mathrm{Ma}$ fu nel Manual de confesores y penitentes di Martín de Azpilcueta, apparso in portoghese nel 1552, tradotto in spagnolo nel 1554 e rielaborato in latino nel 1573 con il titolo di Enchiridion confessariorum, che la materia avrebbe trovato una prima sistemazione a uso del sacramento del perdono. Il dottor Navarro, lettore di Gerson, allievo di Ciruelo e poi collega di Vitoria, ${ }^{51}$ catalogò secondo la nozione di patto implicito i peccati di idolatria, divinazione, sortilegio, maleficio, abuso delle orazioni, delle reliquie, della negromanzia, dell'esorcismo, ricorrendo alla

${ }^{49}$ Francisco de Vitoria, Comentarios a la Secunda Secundae de Santo Tomás, ed. Vicente Beltrán de Heredia (Salamanca: BTE, 1932-1935) V: De iustitia et fortitudine, 44-87; Vitoria, De Arte Magica, 310. Sul Tostado cf. Walter Stephens, Demons Lovers. Witchcraft, Sex, and the Crisis of Belief (Chicago and London: The Chicago University Press, 2002), ad indicem.

${ }^{50} \mathrm{Cf}$. Ioannes de Tabia, Summa summarum quae Tabiena dicitur (Bologna: edibus Benedicti Hectoris, 1567), sub vocibus "Diana" e "Superstitio" [sull'autore cf. Michael Tavuzzi, Renaissance Inquisitors. Dominican Inquisitors and Inquisitorial Districts in Northern Italy, 1474-1527 (Leiden-Boston: Brill, 2007), ad indicem]; Bartolomaeus Fumus, Summa quae Aurea Armilla inscribitur (Piacenza: apud Mutium et Lochetam, 1549), sub voce "Stryigiae" (su Fumi rinvio alla voce da me stilata in Dizionario storico dell'Inquisizione II, 629-30). Sul genere letterario delle summae cf. Miriam Turrini, La coscienza e le leggi. Morale e diritto nei testi per la confessione della prima età moderna (Bologna: il Mulino, 1991). Anche Mazzolini scrisse un'opera a uso dei confessori in cui trattò di superstitio: la Summa Sylvestrina (1515). E così il frate minore Angelo da Chivasso (Summa Angelica, che risale però al 1486).

${ }^{51}$ Per la biografia cf. Vincenzo Lavenia, "Martín Azpilcueta: un profilo," Archivio Italiano per la Storia della Pietà 16 (2003): 15-144. 
Secunda Secundae e al commento del cardinale Tommaso De Vio. E a proposito del volo notturno, fece osservare che incorre nel peccato "qui credit veneficos aut veneficas vel striges corporaliter per aera vehi." Quel mito era una colpa e un'illusione da cancellare; salvo che la constatazione non valeva sempre poiché nel Malleus erano riportate esperienze da ritenere vere "in Germania." Insomma, per quanto i casi fossero "rari," non poteva condannarsi un fedele che confessasse di credervi, ritenendoli però un evento eccezionale. ${ }^{52}$

Era solo un passaggio, inserito in un elenco di superstizioni degne di essere sradicate che meritavano maggiore attenzione disciplinare da parte della Chiesa. E ciò dimostra come una strategia di lotta alla superstitio e di reformatio della religione fosse stata elaborata prima del Tridentino, negli anni in cui fu stilato il Libellus, in nome dell'osservanza del primo comandamento, con molte ambiguità e contraddizioni, ma senza unanimi concessioni alla demonologia. ${ }^{53}$ Quando, nel 1559, il neonato Sant'Uffizio pontificio - negli anni in cui il Concilio di Trento era sospeso per volontà di Paolo IV - si trovò a discutere di un grave caso di stregoneria apertosi a Bologna, il fiscale del tribunale Pietro Belo si proclamò scettico sulla realtà del volo notturno, e per dimostrarlo citò il commento alla Summa del Gaetano. ${ }^{54}$ Ma ciò non impedì né le condanne a morte che seguirono, né che la strategia del-

${ }^{52}$ Cito da Martinus Azpilcueta Navarrus, Opera Omnia in sex tomos distincta, 6 vols. (Venezia: apud Guerilium, 1618-1621) I, 101-06 (Enchiridion confessariorum). Ma cf. una lezione universitaria che il Navarro tenne a Coimbra nel 1548 trattando anche di superstitio: Azpilcueta Navarrus, Opera Omnia IV, 564-70 (Relectio in cap. Novit De judicijs).

${ }^{53} \mathrm{Nel}$ clima dell'emergenza protestante il peccato di superstitio non meritò tuttavia se non un cenno nel Consilium de emendanda Ecclesia elaborato nel 1537 dalla commissione di prelati scelta da Paolo III per indicare le linee di riforma istituzionale da adottare per rispondere alla sfida antiromana. Della commissione fece parte Gasparo Contarini; per i rapporti tra quest'ultimo e gli autori del Libellus sempre utile Hubert Hedin, Contarini und Camaldoli, estratto da Archivio Italiano per la Storia della Pietà, 2 (Roma: Edizioni di Storia e Letteratura, 1953).

${ }^{54}$ Sull'episodio cf. Guido Dall'Olio, “Tribunali vescovili, Inquisizione romana e stregoneria. I processi bolognesi del 1559," in Il piacere del testo. Saggi e studi per Albano Biondi, ed. Adriano Prosperi et al., 2 vol. (Roma: Bulzoni, 2001) I, 63-82. 
la Chiesa cattolica postridentina, dopo gli anni Settanta del XVI secolo, moltiplicasse il numero di processi inquisitoriali e di cause vescovili per sortilegio comminando condanne certo più miti, ma destinate a un numero maggiore di fedeli e di chierici. Fu il trionfo postumo della linea auspicata dal Libellus, si potrebbe dire. Di certo, una scelta disciplinare che fu elaborata prima della Riforma e sarebbe continuata dopo la conclusione del Concilio di Trento.

Vincenzo Lavenia Università di Macerata 
